\title{
Erratum: Electrically driven spin qubit based on valley mixing [Phys. Rev. B 95, 075403 (2017)]
}

Wister Huang, Menno Veldhorst, Neil M. Zimmerman, Andrew S. Dzurak, and Dimitrie Culcer (Received 12 September 2017; published 2 October 2017)

DOI: 10.1103/PhysRevB.96.159901

The labels in Fig. 2 for electric fields were misplaced in the original article, and the correct figure should read
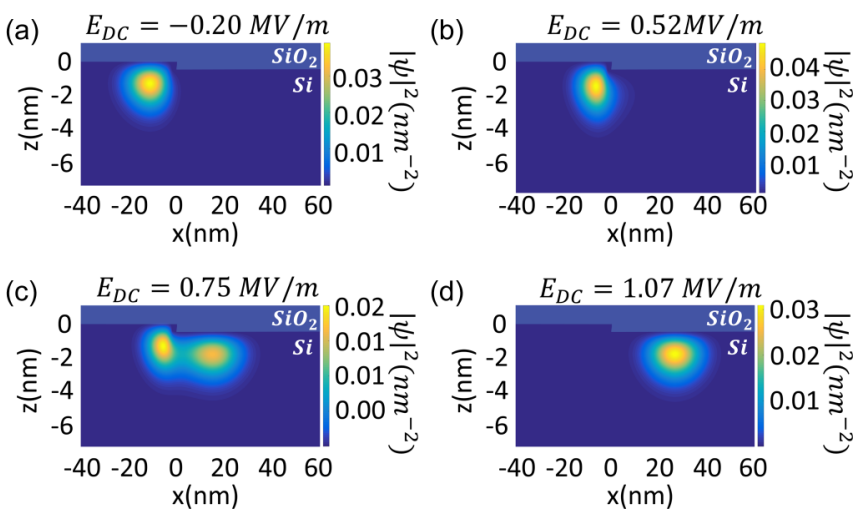

FIG. 1. Evolution of the wave function as it is driven over an interface step. The in-plane electric field is used to drag the wave function over a 5.43- $\AA$ step. During this process (a) the wave function will initially be compressed at the step edge; (b) at higher fields, the electron density starts leaking to the other side of the step; (c) as the wave function pushes against the step, the valley composition becomes more sensitive to the quantum dot position: In the presence of the step, the in-plane electric field can be used to control both $e E_{a c}\langle x\rangle_{01}$ and $\Delta_{v}$, resulting in a significant enhancement of the electron dipole spin resonance frequency. (d) The wave function overcomes the barrier and surmounts the step.

This does not affect the text, the results, the discussion, and the conclusion of the article. 